\title{
APPROXIMATION BY RATIONAL AND MEROMORPHIC FUNCTIONS HAVING A BOUNDED NUMBER OF FREE POLES
}

\author{
BY \\ E. B. $\operatorname{SAFF}\left({ }^{1}\right)$
}

1. Introduction. If a function $g(z)$ defined on a smooth Jordan curve $\Gamma$ of the $z$-plane is the uniform limit on $\Gamma$ of a sequence of polynomials $p_{n}(z)$ of respective degrees $n$, say

$$
\left|g(z)-p_{n}(z)\right| \leqq \varepsilon_{n}(\rightarrow 0), \quad z \text { on } \Gamma,
$$

then one can deduce certain properties of the function $g(z)$ and the sequence $p_{n}(z)$. As a consequence of the Maximum Principle $g(z)$ is the set of boundary values on $\Gamma$ of a function $f(z)$ which is analytic in the interior $D$ of $\Gamma$ and continuous on the closed region $D+\Gamma$. It is also clear that the sequence $p_{n}(z)$ converges to $f(z)$ at each point of $D$. In addition, some continuity properties of $g(z)$ on $\Gamma$ may be deduced if an estimate is known on the rapidity of convergence of the sequence $\varepsilon_{n}$. Indeed, the inequality $\varepsilon_{n} \leqq A / n^{k+\alpha}$, where $k$ is a nonnegative integer and $0<\alpha<1$, implies that the $k$ th derivative of $g(\mathrm{z})$ exists on $\Gamma$ (in the one-dimensional sense) and satisfies there a Lipschitz condition of order $\alpha$.

In this paper we make the weaker assumption that the function $g(z)$ is the uniform limit on $\Gamma$ of a sequence of rational functions each having at most $\nu$ free poles, and we establish analogues of the above mentioned conclusions. Specifically we shall deal with rational functions of type $(n, v)$, i.e., rational functions of the form

$$
r_{n v}(z)=\frac{a_{0} z^{n}+a_{1} z^{n-1}+\cdots+a_{n}}{b_{0} z^{v}+b_{1} z^{v-1}+\cdots+b_{v}}, \quad \sum_{0}^{v}\left|b_{k}\right| \neq 0
$$

for fixed $\nu$.

In $\$ 2$ we show that the condition

$$
\left|g(z)-r_{n v}(z)\right| \leqq \varepsilon_{n}(\rightarrow 0), \quad z \text { on } \Gamma,
$$

implies the existence of a function $f(z)$ which is meromorphic with at most $\nu$ poles in $D$, is continuous on $D+\Gamma$, and is equal to $g(z)$ for $z$ on $\Gamma$. If the function $f(z)$ is known to have precisely $\nu$ poles in $D$, it is further shown that the rational functions

Presented to the Society, November 13, 1967 under the title Approximation by rational functions of type $(n, v)$; received by the editors August $8,1968$.

(1) The results presented here are part of the author's doctoral dissertation, which was directed by Professor J. L. Walsh at the University of Maryland. The research was supported (in part) by N.A.S.A. Fellowship Trainee Grant NsG(t)343. 
$r_{n v}(z)$ must converge to $f(z)$ at each point of $D$. The last result is similar to one obtained by J. L. Walsh [1, p. 3].

In $\$ 3$ and $\S 4$ we establish theorems on the Lipschitz continuity and analyticity of $g(z)$ on $\Gamma$ as a consequence of certain hypotheses on the degree of convergence of the $r_{n v}(z)$ and on the location of the limit points of their poles.

2. Uniform convergence of meromorphic functions. An easy extension of a theorem on polynomial approximation is

THEOREM 1. Let $E$ be a closed bounded point set whose complement is connected and whose interior is nonempty. Suppose $f(z)$ is meromorphic in the interior of $E$ with precisely $\nu$ poles there and is otherwise finite and continuous on $E$. Then there exists a sequence of rational functions $r_{n v}(z)$ of respective types $(n, v)$ which converges uniformly to $f(z)$ on the boundary of $E$.

Proof. Let $q(z)=z^{v}+a_{1} z^{v-1}+\cdots+a_{v}$ be the polynomial of the form indicated having as its zeros the $\nu$ poles of $f(z)$ in the interior of $E$. By a well-known theorem of Mergelyan [2, §A1] the analytic function $q(z) f(z)$ can be uniformly approximated on $E$ as closely as desired by a polynomial, and hence [2, p. 89] there exists a sequence of polynomials $p_{n}(z)$ of respective degrees $n$ which converges uniformly on the boundary of $E$ to $q(z) f(z)$. Theorem 1 now follows by taking $r_{n v}(z)=$ $p_{n}(z) / q(z)$.

If a function $g(z)$ defined merely on the boundary $\partial E$ of $E$ is the uniform limit of polynomials, then as mentioned in $\S 1$ there exists a function $f(z)$ analytic in the interior of $E$ and continuous on $E$ such that $f(z) \equiv g(z)$ for $z$ on $\partial E$. Hence the converse to Theorem 1 is valid for $\nu=0$. To establish a converse result for $\nu>0$ we appeal to the following special case of a result due to $\mathrm{S}$. Warschawski [3]:

THEOREM 2. Let $h(z)$ be analytic in a Jordan region $D_{0}$ and continuous on $D_{0}+\partial D_{0}$. For fixed $\alpha$ on $\partial D_{0}$ let

$$
|h(z)-h(\alpha)| \leqq L|z-\alpha|
$$

hold for all $z$ on $\partial D_{0}$. Then (1) holds for all $z$ on $D_{0}+\partial D_{0}$.

We may now prove

THEOREM 3. Let $D$ be a Jordan region and $g(z)$ a function defined on $\partial D$. Suppose $f_{n}(z)$ is a sequence of functions each meromorphic with at most $v$ poles in $D$ and otherwise finite and continuous on $D+\partial D$. If $\lim _{n \rightarrow \infty} f_{n}(z)=g(z)$ uniformly for $z$ on $\partial D$, then there exists a function $f(z)$ which is meromorphic with at most $v$ poles in $D$ and is otherwise finite and continuous on $D+\partial D$ such that $f(z) \equiv g(z)$ for $z$ on $\partial D$.

Proof. Theorem 3 holds for $\nu=0$, so assume that it holds for $\nu=k-1$ and suppose that each of the functions $f_{n}(z)$ has at most $k$ poles in $D$. Clearly we may assume that each $f_{n}(z)$ has at least one pole in $D$, say at a point $\alpha_{n}$. Let $\alpha$ be a limit point 
of the $\alpha_{n}$ and let $\alpha_{n_{i}}$ be a subsequence which converges to $\alpha$. Then $\left\{\left(z-\alpha_{n_{i}}\right) f_{n_{i}}(z)\right\}$ is a sequence of functions each meromorphic with at most $k-1$ poles in $D$ which converges to the function $(z-\alpha) g(z)$ uniformly for $z$ on $\partial D$. Thus by the induction hypothesis there exists a function $h(z)$ which is meromorphic in $D$ with at most $k-1$ poles there and continuous on $D+\partial D$ such that $h(z)=(z-\alpha) g(z)$ for $z$ on $\partial D$. Set

$$
\begin{aligned}
f(z) & \equiv h(z) /(z-\alpha), & & z \text { in } D, \\
& \equiv g(z), & & z \text { on } \partial D .
\end{aligned}
$$

If $\alpha \in D$, then clearly $f(z)$ is the desired function. If $\alpha \in \partial D$, it remains to show that $f(z)$ is continuous at $\alpha$.

Let $\Gamma_{1}$ be a closed subarc of $\partial D$ which contains the point $\alpha$ and terminates at the distinct points $\beta_{1}$ and $\beta_{2}$, where $\beta_{1} \neq \alpha, \beta_{2} \neq \alpha$. Join the points $\beta_{1}, \beta_{2}$ by an open Jordan arc $\Gamma_{2}$ which lies in $D$, contains no pole of $h(z)$, and is such that $h(z)$ and hence $f(z)$ is analytic in the Jordan region $D_{0}$ bounded by $\Gamma_{1}+\Gamma_{2}$. Since $g(z)$ is continuous on $\Gamma_{1}$ it is bounded there, say by a constant $M$, and so

$$
|h(z)| \leqq M|z-\alpha|
$$

for $z$ on $\Gamma_{1}$. For $M$ large enough inequality (2) also holds for $z$ on $\Gamma_{2}$ since $h(z) /(z-\alpha)$ is finite and continuous on the closure of $\Gamma_{2}$. Theorem 2 thus implies that for an appropriate choice of the constant $M$ inequality (2) is valid for $z$ in $D_{0}$, and hence $f(z)$ is bounded in $D_{0}$. Finally, note that $f(z)$ is continuous on $D_{0}+\Gamma_{1}+\Gamma_{2}-\{\alpha\}$, and that

$$
\lim _{z \rightarrow \alpha: z \in \Gamma_{1}} f(z)=f(\alpha)
$$

and hence the continuity of $f(z)$ at $\alpha$ follows from a theorem of Lindelöf [4, p. 460].

Theorem 3 equivalently states that the family $D(\nu)$ composed of all those functions which are meromorphic with at most $\nu$ poles in $D$ and which are otherwise finite and continuous on $D+\partial D$ is complete with respect to the Tchebycheff (uniform) norm taken over $\partial D$.

Corollary 1. Let $D$ be a Jordan region and let the function $F(z)$ be analytic in $D$ except for a finite number of isolated singularities at the points $z_{1}, \ldots, z_{k}$ in $D$, where at least one $z_{i}$ is an essential singularity of $F(z)$. Suppose $F(z)$ is continous on $D+\partial D-\left\{z_{1}, \ldots, z_{k}\right\}$, and suppose $F_{n}(z)$ is a sequence of functions each meromorphic in $D$ and continuous on $D+\partial D$ which converges uniformly to $F(z)$ on $\partial D$. Then if $p(n)$ denotes the number of poles of $F_{n}(z)$ in $D$, we have $p(n) \rightarrow \infty$ as $n \rightarrow \infty$.

Proof. The contrary assumption implies that there exists an integer $\nu$ and a subsequence $n_{i}$ such that $p\left(n_{i}\right) \leqq \nu$ for $i=1,2, \ldots$. Since the subsequence $F_{n_{i}}(z)$ converges uniformly to $F(z)$ on $\partial D$, Theorem 3 asserts the existence of a function $f(z) \in D(\nu)$ such that $f(z)=F(z)$ for $z$ on $\partial D$. But then $f(z) \equiv F(z)$ for $z$ in $D$, which is absurd. 
We remark that although Corollary 1 is presented here as a consequence of Theorem 3, it may be proved directly from the theory of normal families.

Theorem 1 and Theorem 3 yield

THEOREM 4. Let $\Gamma$ be a Jordan curve with interior $D$ and let $g(z)$ be a function defined (finite) on $\Gamma$. A necessary and sufficient condition that $g(z)$ be the set of boundary values on $\Gamma$ of a function which is meromorphic with at most $v$ poles in $D$ and which is otherwise finite and continuous on $D+\Gamma$ is that there exist a sequence of rational functions $r_{n v}(z)$ of respective types $(n, v)$ such that $\lim _{n \rightarrow \infty} r_{n v}(z)=g(z)$ uniformly for $z$ on $\Gamma$.

If the function $g(z)$ is the set of boundary values of a meromorphic function $f(z)$ known to have precisely $\nu$ poles in $D$, then any sequence of rational functions of respective types $(n, v)$ which converges uniformly to $g(z)$ on $\Gamma$ necessarily converges to $f(z)$ in $D$. More generally we prove

THEOREM 5. Suppose $f_{0}(z)$ is meromorphic with precisely $\nu$ poles in a bounded domain $D$ and is otherwise finite and continuous on $D+\partial D$. If $f_{n}(z)$ is a sequence of functions each meromorphic with at most $\nu$ poles in $D$ and continuous on $D+\partial D$ which satisfies

$$
\left|f_{0}(z)-f_{n}(z)\right| \leqq \varepsilon_{n}(\rightarrow 0), \quad z \text { on } \partial D
$$

then:

(i) For $n$ sufficiently large each $f_{n}(z)$ has precisely $v$ poles in $D$, and these poles approach respectively the $\nu$ poles of $f_{0}(z)$ in $D$.

(ii) The $f_{n}(z)$ converge to $f_{0}(z)$ in the domain $D^{\prime}$ obtained from $D$ by deleting the $\nu$ poles of $f_{0}(z)$.

(iii) For each closed set $S \subset D^{\prime}$ we have for $n$ large

$$
\left[\max \left|f_{0}(z)-f_{n}(z)\right| ; z \text { on } S\right] \leqq M(S) \varepsilon_{n},
$$

where $M(S)$ is a constant dependent only on $S, D$, and on the sequence $f_{n}(z)$.

Proof. For $n=0,1,2, \ldots$, let $q_{n}(z)=z^{\mu_{n}}+\cdots+a_{n}$ denote the polynomial of the form indicated having as its zeros the poles of $f_{n}(z)$ in $D$. Since $D$ is bounded and since $\mu_{n} \leqq \nu$ for each $n$, the sequence $q_{n}(z)$ is uniformly bounded in $D$. A wellknown application of Lagrange's Interpolation Formula thus implies that the $q_{n}(z)$ form a normal family in the finite plane and that each limit function of the family is a polynomial of the form $z^{\mu}+\cdots+a, 0 \leqq \mu \leqq \nu$.

Let $q(z)$ be any such limit function and $q_{n_{i}}(z)$ a subsequence which converges uniformly to $q(z)$ on compact sets of the plane. From (3) we have

$$
\lim _{i \rightarrow \infty} q_{n_{i}}(z) f_{n_{i}}(z)=q(z) f_{0}(z),
$$

uniformly for $z$ on $\partial D$, and so the analyticity of the functions $q_{n_{i}}(z) f_{n_{t}}(z)$ implies that $q(z) f_{0}(z)$ is analytic in $D$. Hence the polynomial $q_{0}(z)$ must be a factor of 
$q(z)$; and since $q(z)$ is monic and has at most $\nu$ zeros, it follows that $q(z) \equiv q_{0}(z)$. Thus the only limit function of the $q_{n}(z)$ is $q_{0}(z)$, and hence the sequence $q_{n}(z)$ converges to $q_{0}(z)$ uniformly on compact sets of the plane. Conclusion (i) now follows from Hurwitz's Theorem.

Now let $S \subset D^{\prime}$ be closed. Since the $q_{n}(z)$ are uniformly bounded on $\partial D$ we obtain from (3)

$$
\left|q_{0}(z) q_{n}(z) f_{0}(z)-q_{0}(z) q_{n}(z) f_{n}(z)\right| \leqq A \varepsilon_{n}, \quad z \text { on } \partial D .
$$

The function whose absolute value appears in (4) is analytic in $D$, and so (4) holds for $z$ on $S$. By conclusion (i) the set $S$ contains no limit points of the poles of the $f_{n}(z)$ and hence for $n$ large enough we have $\left|q_{n}(z) q_{0}(z)\right| \geqq m>0$ for $z$ on $S$. There follows

$$
\left|f_{0}(z)-f_{n}(z)\right| \leqq A \varepsilon_{n} / m, \quad z \text { on } S,
$$

which completes the proof of Theorem 5 .

From conclusion (i) we deduce

COROLlaRY 2. If $f_{0}(z)$ and $D$ are as in Theorem 5 and $f_{n}(z)$ is a sequence of functions each meromorphic with at most $\mu(<\nu)$ poles in $D$ and continuous on $D+\partial D$, then the $f_{n}(z)$ do not converge uniformly to $f_{0}(z)$ on $\partial D$.

The assumption that the number of poles of the functions $f_{n}(z)$ not exceed the number of poles of the limit function $f_{0}(z)$ cannot be weakened in Theorem 5 . Indeed the sequence $f_{n}(z) \equiv(z-1+1 / n) / z(z-1)$ converges uniformly to $1 / z$ on $|z|=2$, but does not converge to $1 / z$ for $z=1$. The method of proof of Theorem 5 does however yield

Corollary 3. Suppose $f_{0}(z)$ and $D$ are as in Theorem 5 and $f_{n}(z)$ is a sequence of functions each meromorphic with at most $\eta$ poles in $D$ and continuous on $D+\partial D$. If $\lim _{n \rightarrow \infty} f_{n}(z)=f_{0}(z)$ uniformly for $z$ on $\partial D$, then each pole of $f_{0}(z)$ in $D$ is a limit point of poles of the $f_{n}(z)$, and $\lim _{n \rightarrow \infty} f_{n}(z)=f_{0}(z)$ uniformly on each closed subset of $D$ which contains no limit points of the poles of the $f_{n}(z)$.

An easy generalization of Hurwitz's Theorem is

COROLlary 4. Suppose, in addition to the hypotheses of Theorem 5, that the function $f_{0}(z)$ does not vanish on $\partial D$. Then for $n$ sufficiently large $f_{n}(z)$ and $f_{0}(z)$ have the same number of zeros in $D$.

The proof of Corollary 4 is left to the reader.

If the function $f_{0}(z)$ of Theorem 5 has a pole at a point $\alpha$ in $D$, then we can apply Corollary 4 to obtain an estimate on the degree of divergence of the sequence $f_{n}(\alpha)$. We choose a constant $\delta(>0)$ so small that $f_{0}(z)$ is analytic and nonzero in $0<|z-\alpha| \leqq \delta$. From conclusion (iii) of Theorem 5 there follows

$$
\left|1 / f_{0}(z)-1 / f_{n}(z)\right| \leqq M \varepsilon_{n}, \quad|z-\alpha|=\delta .
$$


Since for $n$ sufficiently large $f_{n}(z)$ and $f_{0}(z)$ have the same number of poles in $|z-\alpha| \leqq \delta$, Corollary 4 implies that the $f_{n}(z)$ do not vanish there for $n$ large. Hence inequality (5) holds for $z=\alpha$, and so $\left|f_{n}(\alpha)\right| \geqq 1 / M \varepsilon_{n}$.

Theorem 3 and Theorem 5 yield the following dual theorems which are obtained by interchanging the poles and zeros of the functions $f_{n}(z)$ :

THEOREM 6. Let $D$ be a Jordan region and let $G(z)$ be defined and different from zero on $\partial D$. Suppose $F_{n}(z)$ is a sequence of functions each meromorphic with at most $\nu$ zeros in $D$, continuous on $D+\partial D$, and finite on $\partial D$. If $\lim _{n \rightarrow \infty} F_{n}(z)=G(z)$ uniformly for $z$ on $\partial D$, then $G(z)$ is the set of boundary values on $\partial D$ of a function which is meromorphic with at most $\nu$ zeros in $D$ and continuous on $D+\partial D$.

THEOREM 7. Suppose the function $F_{0}(z)$ is meromorphic with precisely $v$ zeros in a bounded domain $D$, and is continuous on $D+\partial D$ and finite and different from zero on $\partial D$. If $F_{n}(z)$ is a sequence of functions each meromorphic with at most $\nu$ zeros in $D$ and continuous on $D+\partial D$ which satisfies

$$
\left|F_{0}(z)-F_{n}(z)\right| \leqq \varepsilon_{n}(\rightarrow 0), \quad z \text { on } \partial D,
$$

then:

(i) For $n$ sufficiently large each $F_{n}(z)$ has precisely $v$ zeros in $D$, and these zeros approach respectively the $\nu$ zeros of $F_{0}(z)$ in $D$.

(ii) Each pole of $F_{0}(z)$ in $D$ is a limit point of poles of the $F_{n}(z)$, multiplicity included, and the $F_{n}(z)$ have no other limit point of poles in $D$.

(iii) $\operatorname{Lim}_{n \rightarrow \infty} F_{n}(z)=F_{0}(z)$ uniformly on each closed set $S \subset D$ which contains no poles of $F_{0}(z)$, and for $n$ large

$$
\left[\max \left|F_{0}(z)-F_{n}(z)\right| ; z \text { on } S\right] \leqq M(S) \varepsilon_{n},
$$

where $M(S)$ is a constant dependent only on $S, D$, and on the sequence $F_{n}(z)$.

The proofs of Theorem 6 and Theorem 7, which follow from methods used by J. L. Walsh [5], are left to the reader.

3. Lipschitz continuity. We now apply the results of $\S 2$ to obtain theorems which relate the boundary continuity of a meromorphic function $f(z)$ to the degree of approximation of $f(z)$ by rational functions.

Let $\Gamma$ be an analytic Jordan curve and $D$ its interior. We say that a function $f(z)$ belongs to class $L_{\nu}(k, \alpha)$ on $\Gamma$, where $\nu$ and $k$ are nonnegative integers and $0<\alpha<1$, if $f(z)$ is meromorphic with at most $\nu$ poles in $D$ and is otherwise finite and continuous on $D+\Gamma$, and if $f^{(k)}(z)$ exists on $\Gamma$ in the one-dimensional sense and satisfies there a Lipschitz condition of order $\alpha$, i.e.,

$$
\left|f^{(k)}\left(z_{1}\right)-f^{(k)}\left(z_{2}\right)\right| \leqq L\left|z_{1}-z_{2}\right|^{\alpha}, \quad z_{1}, z_{2} \text { on } \Gamma \text {, }
$$

where $L$ is a constant independent of $z_{1}$ and $z_{2}$.

It is of importance to mention here that the property of a function that it has a $k$ th derivative satisfying condition (6) is invariant under conformal mapping. This fact is well-illustrated by the following theorem [6, p. 24]: 
THEOREM 8. Let the function $g(z)$ be defined on an analytic Jordan curve $\Gamma$. A necessary and sufficient condition that $g(z)$ possess a kth derivative on $\Gamma$ which satisfies a Lipschitz condition of order $\alpha(0<\alpha<1)$ on $\Gamma$ is that there exist a region $D_{1}$ containing $\Gamma$ and a sequence of functions $f_{n}(z)$ analytic in $D_{1}$ and satisfying

$$
\begin{aligned}
\left|f_{n}(z)\right| & \leqq A R^{n}, & & z \text { in } D_{1}, \\
\left|g(z)-f_{n}(z)\right| & \leqq A_{1} / n^{k+\alpha}, & & z \text { on } \Gamma .
\end{aligned}
$$

The fundamental theorem relating the degree of best polynomial approximation on $\Gamma$ to the existence of functions of class $L_{0}(k, \alpha)$ on $\Gamma$ was established by $\mathbf{J} . \mathbf{H}$. Curtiss, W. E. Sewell, and J. L. Walsh [6, p. 27] and is stated as

THEOREM 9. Let $\Gamma$ be an analytic Jordan curve and $f(z)$ a function defined on $\Gamma$. Then the following statements are equivalent:

(i) $f(z)$ is the set of boundary values on $\Gamma$ of a function of class $L_{0}(k, \alpha)$ on $\Gamma$.

(ii) There exists a sequence of polynomials $p_{n}(z)$ of respective degrees $n$ such that

$$
\left|f(z)-p_{n}(z)\right| \leqq A / n^{k+\alpha}, \quad z \text { on } \Gamma .
$$

(iii) There exists a domain $D_{0}$ containing $\Gamma$ and its interior and a sequence of functions $f_{n}(z)$ analytic in $D_{0}$ satisfying the inequalities

$$
\begin{aligned}
\left|f_{n}(z)\right| & \leqq A_{1} R^{n}, & & z \text { in } D_{0}, \\
\left|f(z)-f_{n}(z)\right| & \leqq A_{2} / n^{k+\alpha}, & & z \text { on } \Gamma .
\end{aligned}
$$

An extension of Theorem 9 to the case $\nu>0$ is given in

THEOREM 10. Let $\Gamma$ be an analytic Jordan curve and $D$ its interior. If $f(z)$ is meromorphic in $D$ with precisely $\nu$ poles there and is otherwise finite and continuous on $D+\Gamma$, then the following statements are equivalent:

(i) $f(z)$ belongs to class $L_{v}(k, \alpha)$ on $\Gamma$.

(ii) There exists a sequence of rational functions $r_{n v}(z)$ of respective types $(n, \nu)$ such that

$$
\left|f(z)-r_{n v}(z)\right| \leqq A / n^{k+\alpha}, \quad z \text { on } \Gamma .
$$

(iii) There exists a domain $D_{0}$ containing $D+\Gamma$ and a sequence of meromorphic functions $f_{n}(z)$ of the form

$$
f_{n}(z)=f_{n 1}(z) / f_{n 2}(z),
$$

where $f_{n 1}(z)$ is analytic in $D_{0}$ and $f_{n 2}(z)$ is a polynomial of the form $z^{\lambda}+a_{1} z^{\lambda-1}$ $+\cdots+a_{\lambda}, 0 \leqq \lambda \leqq \nu$, such that the following inequalities hold:

$$
\begin{aligned}
\left|f_{n 1}(z)\right| & \leqq A_{1} R^{n}, & & z \text { in } D_{0}, \\
\left|f(z)-f_{n}(z)\right| & \leqq A_{2} / n^{k+\alpha}, & & z \text { on } \Gamma .
\end{aligned}
$$


Proof. Suppose $f(z) \in L_{v}(k, \alpha)$ on $\Gamma$, and let $q(z)$ be the monic polynomial of degree $\nu$ whose zeros are the poles of $f(z)$ in $D$. It is easy to see that $q(z) f(z) \in L_{0}(k, \alpha)$ on $\Gamma$, and hence there exists a sequence of polynomials $p_{n}(z)$ of respective degrees $n$ such that

$$
\left|q(z) f(z)-p_{n}(z)\right| \leqq B / n^{k+\alpha}, \quad z \text { on } \Gamma
$$

Whence

$$
\left|f(z)-p_{n}(z) / q(z)\right| \leqq A / n^{k+\alpha}, \quad z \text { on } \Gamma,
$$

and so (i) implies (ii).

Now assume (ii) holds and write $r_{n v}(z)=P_{n}(z) / Q_{n}(z)$, where $P_{n}(z)$ is a polynomial of degree $n$ and $Q_{n}(z)$ is the polynomial of the form $z^{\mu}+\cdots+a, 0 \leqq \mu \leqq \nu$, whose zeros are the finite poles of $r_{n v}(z)$. Theorem 5 implies that the $Q_{n}(z)$ are uniformly bounded on $\Gamma$, and since the $r_{n v}(z)$ are also uniformly bounded there, the same must be true of the $P_{n}(z)$. Thus by the Generalized Bernstein Lemma [2, p. 77] we deduce for $z$ on any bounded domain $D_{0},\left|P_{n}(z)\right| \leqq A_{1} R^{n}$.

It remains to show that (iii) implies (i). The uniform convergence of the $f_{n}(z)$ on $\Gamma$ implies, by Theorem 5 , that the zeros of the $f_{n 2}(z)$ approach the poles of $f(z)$ in $D$. Hence there exists an annular region $D_{1}$ containing $\Gamma$ such that for $n$ sufficiently large each $f_{n}(z)$ is analytic in $D_{1}$ and satisfies there the inequality $\left|f_{n}(z)\right| \leqq A_{3} R^{n}$. Statement (i) now follows from Theorem 8 .

Theorem 10, in contrast with Theorem 9, assumes not merely that $f(z)$ be defined on $\Gamma$, but that $f(z)$ be the boundary values on $\Gamma$ of a meromorphic function known to have precisely $\nu$ poles interior to $\Gamma$. This hypothesis can be weakened by assuming, instead, that all the finite poles of the rational functions $r_{n v}(z)$ lie in $D$. In the proof of such a result it is convenient to have for reference

LEMMA 1. Suppose $f(z)$ is meromorphic in $U:|z|<1$ with precisely $\mu(\geqq 0)$ poles there, and is otherwise finite and continuous on $|z| \leqq 1$. If $\alpha_{1}, \alpha_{2}, \ldots, \alpha_{\mu}$ are the poles of $f(z)$ in $U$ and if $r(z)$ is a rational function of type $(n, v), n \geqq v$, having all its finite poles in $U$, then there exists a rational function $R(z)$ of the form

such that

$$
R(z)=\frac{c_{0} z^{n+\mu}+c_{1} z^{n+\mu-1}+\cdots+c_{n+\mu}}{\left(z-\alpha_{1}\right)\left(z-\alpha_{2}\right) \cdots\left(z-\alpha_{\mu}\right)}
$$

$$
[\max |f(z)-R(z)| ;|z|=1] \leqq 2^{v}[\max |f(z)-r(z)| ;|z|=1] .
$$

Proof. If $r(z)$ has no finite pole, we simply take $R(z) \equiv r(z)$. Otherwise let $\beta_{1}, \beta_{2}, \ldots, \beta_{\lambda}$ be the finite poles of $r(z)$ and let

$$
T(z) \equiv \prod_{i=1}^{\mu}\left(z-\alpha_{i}\right) /\left(1-\bar{\alpha}_{i} z\right), \quad B(z) \equiv T(z) \prod_{i=1}^{\lambda}\left(z-\beta_{i}\right) /\left(1-\bar{\beta}_{i} z\right) .
$$

We note that $S_{0}(z) \equiv B(z) r(z)$ is a rational function of the form

$$
S_{0}(z)=q_{0}(z) /\left(1-\bar{\alpha}_{1} z\right) \cdots\left(1-\bar{\alpha}_{\mu} z\right)\left(1-\bar{\beta}_{1} z\right) \cdots\left(1-\bar{\beta}_{\lambda} z\right),
$$


where $q_{0}(z)$ is a polynomial of degree $n+\mu$. Setting $M \equiv[\max |f(z)-r(z)| ;|z|=1]$ we obtain from the Maximum Principle

$$
\left|B(z) f(z)-S_{0}(z)\right| \leqq M, \quad|z| \leqq 1 .
$$

Since $B\left(\beta_{\lambda}\right) f\left(\beta_{\lambda}\right)=0$, the triangle inequality yields

$$
\left|B(z) f(z)-\left(S_{0}(z)-S_{0}\left(\beta_{\lambda}\right)\right)\right| \leqq 2 M, \quad|z|=1,
$$

and hence

$$
\left|\left[\left(1-\bar{\beta}_{\lambda} z\right) /\left(z-\beta_{\lambda}\right)\right] B(z) f(z)-S_{1}(z)\right| \leqq 2 M, \quad|z|=1,
$$

where $S_{1}(z) \equiv\left(S_{0}(z)-S_{0}\left(\beta_{\lambda}\right)\right)\left(1-\beta_{\lambda} z\right) /\left(z-\beta_{\lambda}\right)$. Note that $S_{1}(z)$ is a rational function of the form

$$
S_{1}(z)=q_{1}(z) /\left(1-\bar{\alpha}_{1} z\right) \cdots\left(1-\bar{\alpha}_{\mu} z\right)\left(1-\bar{\beta}_{1} z\right) \cdots\left(1-\bar{\beta}_{\lambda-1} z\right),
$$

where $q_{1}(z)$ is a polynomial of degree $n+\mu$.

Since inequality (9) holds for $|z| \leqq 1$, the same reasoning used to deduce (9) as a consequence of $(8)$ yields

$$
\left|\left[\frac{1-\beta_{\lambda-1} z}{z-\beta_{\lambda-1}}\right]\left[\frac{1-\beta_{\lambda} z}{z-\beta_{\lambda}}\right] B(z) f(z)-S_{2}(z)\right| \leqq 4 M, \quad|z|=1,
$$

where $S_{2}(z)$ is a rational function of the form

$$
S_{2}(z)=q_{2}(z) /\left(1-\bar{\alpha}_{1} z\right) \cdots\left(1-\bar{\alpha}_{\mu} z\right)\left(1-\bar{\beta}_{1} z\right) \cdots\left(1-\bar{\beta}_{\lambda-2} z\right),
$$

and $q_{2}(z)$ is a polynomial of degree $n+\mu$.

After $\lambda$ steps we obtain a polynomial $q_{\lambda}(z)$ of degree $n+\mu$ which satisfies

$$
\left|T(z) f(z)-\frac{q_{\lambda}(z)}{\left(1-\bar{\alpha}_{1} z\right) \cdot\left(1-\bar{\alpha}_{\mu} z\right)}\right| \leqq 2^{\lambda} M \leqq 2^{v} M, \quad|z|=1,
$$

and so Lemma 1 follows by taking $R(z) \equiv q_{\lambda}(z) /\left(z-\alpha_{1}\right) \cdots\left(z-\alpha_{\mu}\right)$.

We may now prove

THEOREM 11. Let $g(z)$ be a function defined (finite) on an analytic Jordan curve $\Gamma$ with interior $D$. Then the following statements are equivalent:

(i) $g(z)$ is the set of boundary values on $\Gamma$ of a function which belongs to class $L_{v}(k, \alpha)$ on $\Gamma$.

(ii) There exists a sequence of rational functions $r_{n v}(z)$ of respective types $(n, v)$ having all their finite poles in $D$ and satisfying

$$
\left|g(z)-r_{n v}(z)\right| \leqq A / n^{k+\alpha}, \quad z \text { on } \Gamma .
$$

(iii) There exists a domain $D_{0}$ containing $D+\Gamma$ and a sequence of meromorphic functions $f_{n}(z)$ of the form (7), where all the zeros of $f_{n 2}(z)$ lie in $D$, such that

$$
\begin{aligned}
\left|f_{n 1}(z)\right| & \leqq A_{1} R^{n}, & & z \text { in } D_{0}, \\
\left|g(z)-f_{n}(z)\right| & \leqq A_{2} / n^{k+\alpha}, & & z \text { on } \Gamma .
\end{aligned}
$$


Proof. That (i) implies (ii) and (iii) is immediate from Theorem 10. Therefore since (ii) clearly implies (iii), we need only show (iii) implies (i).

Assuming statement (iii) holds, Theorem 3 asserts the existence of a function $f(z)$ which is meromorphic with precisely $\mu(\leqq \nu)$ poles in $D$, is continuous on $D+\Gamma$, and equal to $g(z)$ for $z$ on $\Gamma$. Let $z=\psi(w)$ map $U:|w|<1$ conformally onto $D$, and set $F(w) \equiv f(\psi(w))$. We shall prove $F(w) \in L_{v}(k, \alpha)$ on $C:|w|=1$, which is equivalent to statement (i).

Since $\Gamma$ is analytic, there exists a constant $\rho(>1)$ such that $\psi(w)$ is analytic on $|w| \leqq \rho$ and such that the image of $|w| \leqq \rho$ under $z=\psi(w)$ lies in $D_{0}$. From (10) and the fact that all the zeros of $f_{n 2}(z)$ lie in $D$ we have

$$
\left|f_{n}(\psi(w))\right| \leqq M R^{n}, \quad w \text { on } C_{\rho}:|w|=\rho .
$$

The function $s_{n}(w) \equiv f_{n}(\psi(w))$ is analytic on $|w| \leqq \rho$ except for a finite number of poles, say at the points $\beta_{n 1}, \beta_{n 2}, \ldots, \beta_{n, \lambda(n)}$. Since $\psi(w)$ is schlicht and since all the poles of $f_{n}(z)$ lie in $D$, it follows that $\lambda(n) \leqq \nu$, and that $\left|\beta_{n j}\right|<1$ for $j=1,2, \ldots, \lambda(n)$.

Now let $P_{n, N}(w)$ be the polynomial in $w$ of degree $N+\lambda(n)-1$ which interpolates to the analytic function

$$
S_{n}(w) \equiv s_{n}(w)\left(w-\beta_{n 1}\right)\left(w-\beta_{n 2}\right) \cdots\left(w-\beta_{n, \lambda(n)}\right)
$$

in the points $\beta_{n 1}, \beta_{n 2}, \ldots, \beta_{n, \lambda(n)}$ and in the origin taken of multiplicity $N$. The Hermite Interpolation Formula asserts

$$
S_{n}(w)-P_{n, N}(w)=\frac{1}{2 \pi i} \int_{C_{\rho}} \frac{\left(w-\beta_{n 1}\right) \cdots\left(w-\beta_{n, \lambda(n)}\right) w^{N} S_{n}(t)}{\left(t-\beta_{n 1}\right) \cdots\left(t-\beta_{n, \lambda(n)}\right) t^{N}(t-w)} d t
$$

for $w$ on $C$, and so

$$
s_{n}(w)-\frac{P_{n, N}(w)}{\left(w-\beta_{n 1}\right) \cdots\left(w-\beta_{n, \lambda(n)}\right)}=\frac{1}{2 \pi i} \int_{C_{\rho}} \frac{w^{N} s_{n}(t)}{t^{N}(t-w)} d t,
$$

for $w$ on $C$. Thus from (12) we deduce

$$
\left|s_{n}(w)-T_{n, N}(w)\right| \leqq M_{1} R^{n} / \rho^{N}, \quad w \text { on } C,
$$

where $T_{n, N}(w) \equiv P_{n, N}(w) /\left(w-\beta_{n 1}\right) \cdots\left(w-\beta_{n, \lambda(n)}\right)$.

Now choose a positive integer $\tau$ so large that $\gamma \equiv R / \rho^{\tau}<1$. Then

$$
\left|s_{n}(w)-T_{n, \tau n}(w)\right| \leqq M_{1} \gamma^{n}, \quad w \text { on } C,
$$

and hence from (11) and the triangle inequality there follows

$$
\left|F(w)-T_{n, \tau n}(w)\right| \leqq A_{2} / n^{k+\alpha}+M_{1} \gamma^{n} \leqq A_{3} / n^{k+\alpha},
$$

for $w$ on $C$. We note that $T_{n, \tau n}(w)$ is a rational function of type $(\tau n+\nu-1, \nu)$ having all its finite poles in $U$. Thus since $F(w)$ is meromorphic with precisely $\mu$ poles in $U$ and is continuous on $|w| \leqq 1$, Lemma 1 implies that there exists a sequence of rational functions $R_{n}(w)$ of respective types $(\tau n+\nu-1+\mu, \mu)$ satisfying

$$
\left|F(w)-R_{n}(w)\right| \leqq A_{4} / n^{k+\alpha}, \quad w \text { on } C,
$$


and having all their finite poles on a closed set interior to $C$. It is easy to see that for $\delta(>0)$ sufficiently small we have

$$
\left|R_{n}(w)\right| \leqq M_{2}\left(\rho^{\tau}\right)^{n}, \quad 1-\delta<|w|<\rho,
$$

and so Theorem 8 implies $F(w) \in L_{v}(k, \alpha)$ on $C$, which completes the proof.

Theorem 11 and $[2, \S 9.7$, Lemma I] yield

Corollary 5. Suppose $g(z)$ is defined on an analytic Jordan curve $\Gamma$ and $r_{n v}(z)$ is a sequence of rational functions of respective types $(n, v)$ satisfying

$$
\left|g(z)-r_{n v}(z)\right| \leqq A / n^{k+\alpha}, \quad z \text { on } \Gamma,
$$

where $k$ is a nonnegative integer and $0<\alpha<1$. If no point of $\Gamma$ is a limit point of those poles of the $r_{n v}(z)$ which lie exterior to $\Gamma$, then the kth derivative of $g(z)$ exists on $\Gamma$ and satisfies a Lipschitz condition of order $\alpha$ there.

In the theorems of this section the case $\alpha=1$ is excluded. However, Theorem 9 holds [7] if the Lipschitz condition of order unity on $\Gamma$ is replaced by the Zygmund condition

$$
|f(x+h)+f(x-h)-2 f(x)| \leqq L|h|,
$$

with respect to arc length on $\Gamma$. The extensions of Theorem 10 and Theorem 11 to this exceptional case are immediate.

4. Overconvergence. The theorems of $\S 2$ and $\S 3$ dealt with approximation to a function meromorphic interior to a closed curve $\Gamma$ and continuous on $\Gamma$. We turn now to the questions of analyticity on $\Gamma$ and its relationship to the overconvergence of sequences of rational functions. The term overconvergence is here meant to describe the phenomenon that certain sequences which converge sufficiently rapidly on $\Gamma$ necessarily converge on a point set containing $\Gamma$ in its interior.

Of fundamental importance in the study of overconvergence of sequences of rational functions of type $(n, v)$ is a lemma [8] due to J. L. Walsh. We state this result in the following slightly more general form:

LEMMA 2. Let $E$, with boundary $\Gamma$, be a closed bounded point set whose complement (with respect to the extended plane) $K$ is connected and regular in the sense that $K$ possesses a Green's function $G(z)$ with pole at infinity. Let $\Gamma_{\sigma}(\sigma>1)$ denote generically the locus $G(z)=\log \sigma$, and suppose that rational functions $r_{n v}(z)$ of respective types. $(n, v)$ satisfy the inequality

$$
\limsup _{n \rightarrow \infty}\left[\max \left|r_{n \nu}(z)\right| ; z \text { on } \Gamma\right]^{1 / n} \leqq 1 / \rho, \quad 1<\rho \leqq \infty .
$$

Let $S$ be a closed set in the interior of $\Gamma_{\sigma}, 1<\sigma<\rho$, and containing no limit point of the poles of the $r_{n v}(z)$. Then the sequence $r_{n v}(z)$ converges uniformly to zero on $S$, and we have

$$
\limsup _{n \rightarrow \infty}\left[\max \left|r_{n v}(z)\right| ; z \text { on } S\right]^{1 / n} \leqq \sigma / \rho .
$$

The $r_{n v}(z)$ need not be defined for every $n$. 
Because of the lemma's frequent use we submit a new and brief

Proof. Let $\sigma<\mu<\infty$ and let $q_{n}(z)$ be the polynomial of the form $q_{n}(z)=z^{\lambda n}$ $+\cdots+a_{\lambda_{n}}, 0 \leqq \lambda_{n} \leqq v$, whose zeros are those poles of $r_{n v}(z)$ which lie interior to $\Gamma_{\mu}$. We note that the function $s_{n}(z) \equiv q_{n}(z) r_{n v}(z)$ is a rational function of type $(n, n)$ whose poles lie on or exterior to $\Gamma_{\mu}$. From (13) and the uniform boundedness of the $q_{n}(z)$ on $\Gamma$ we have

$$
\limsup _{n \rightarrow \infty}\left[\max \left|s_{n}(z)\right| ; z \text { on } E\right]^{1 / n} \leqq 1 / \rho
$$

and so from $[2, \S 9.7$, Lemma I] there follows

$$
\limsup _{n \rightarrow \infty}\left[\max \left|s_{n}(z)\right| ; z \text { on } S\right]^{1 / n} \leqq(\mu \sigma-1) /(\mu-\sigma) \rho .
$$

Since $S$ contains no limit point of the poles of the $r_{n v}(z)$, the functions $q_{n}(z)$ are for $n$ sufficiently large uniformly bounded below in modulus by a positive constant on $S$ and hence

$$
\limsup _{n \rightarrow \infty}\left[\max \left|r_{n v}(z)\right| ; z \text { on } S\right]^{1 / n} \leqq(\mu \sigma-1) /(\mu-\sigma) \rho .
$$

Letting $\mu \rightarrow \infty$ we obtain (14).

An easy application of Lemma 2 to analytic continuation is

THEOREM 12. Let $E, \Gamma$, and $\Gamma_{\sigma}$ be as in Lemma 2 and let $E_{\sigma}$ denote the interior of $\Gamma_{\sigma}$. Suppose that $g(z)$ is a function defined (finite) on $\Gamma$ and $r_{n v}(z)$ is a sequence of rational functions of respective types $(n, v)$ which satisfy the inequality

$$
\limsup _{n \rightarrow \infty}\left[\sup \left|g(z)-r_{n \nu}(z)\right| ; z \text { on } \Gamma\right]^{1 / n} \leqq 1 / \rho<1 \text {. }
$$

If no point of $\Gamma_{\rho}$ is a limit point of those poles of the $r_{n v}(z)$ which lie in $E_{\rho}$, then there exists a function $f(z)$ which is meromorphic with at most $v$ poles in $E_{\rho}$ such that $f(z) \equiv g(z)$ for $z$ on $\Gamma$.

Proof. Set $t_{n}(z) \equiv r_{n v}(z)-r_{n-1, v}(z)$, and note that the $t_{n}(z)$ form a sequence of rational functions of respective types $(n+\nu, 2 v)$ which satisfies

$$
\limsup _{n \rightarrow \infty}\left[\max \left|t_{n}(z)\right| ; z \text { on } \Gamma\right]^{1 / n} \leqq 1 / \rho .
$$

For $\varepsilon>0$ sufficiently small, none of the poles of the $r_{n v}(z)$ and hence of the $t_{n}(z)$ lie on $\Gamma_{\rho-\varepsilon}$. Thus from Lemma 2 we deduce

$$
\limsup _{n \rightarrow \infty}\left[\max \left|t_{n}(z)\right| ; z \text { on } \Gamma_{\rho-\varepsilon}\right]^{1 / n} \leqq(\rho-\varepsilon) / \rho,
$$

which implies that the $r_{n v}(z)$ are uniformly bounded on $\Gamma_{\rho-\varepsilon}$.

Now write $r_{n v}(z)=h_{n}(z) / q_{n}(z)$, where $h_{n}(z)$ is analytic in $E_{\rho-\varepsilon}$ and $q_{n}(z)$ is the monic polynomial whose zeros are those poles of $r_{n v}(z)$ which lie in $E_{\rho-\varepsilon}$. The 
uniform boundedness of the $r_{n v}(z)$ and the $q_{n}(z)$ on $\Gamma_{\rho-\varepsilon}$ implies that the $h_{n}(z)$ form a normal family in $E_{\rho-\varepsilon}$. Thus there exists a subsequence $s_{k}(z)$ of the $r_{n \nu}(z)$ and a function $f(z)$ meromorphic with at most $\nu$ poles in $E_{\rho-\varepsilon}$ such that $\lim _{k \rightarrow \infty} s_{k}(z)$ $=f(z)$ uniformly on each closed subset of an open set $D$ obtained from $E_{\rho-\varepsilon}$ by the omission of at most $\nu$ points. Clearly the identity $f(z)=g(z)$ holds with at most $\nu$ exceptions for $z$ on $\Gamma$, and hence by the continuity of $f(z)$ and $g(z)$ on $\Gamma$ the identity holds everywhere on $\Gamma$. Theorem 12 now follows from the arbitrariness of $\varepsilon$.

COROLlaRY 6. With the geometric conditions of Lemma 2 suppose that the function $F(z)$ is meromorphic with precisely $\nu$ poles in the interior $T$ of $E$ and is otherwise finite and continuous on $E$. If there exists a sequence of rational functions $r_{n v}(z)$ of respective types $(n, \nu)$ satisfying

$$
\limsup _{n \rightarrow \infty}\left[\max \left|F(z)-r_{n v}(z)\right| ; z \text { on } \Gamma\right]^{1 / n} \leqq 1 / \rho<1,
$$

then $F(z)$ can be extended so as to be analytic on $E_{\rho}-T$.

Proof. By Theorem 5 the sequence $r_{n v}(z)$ converges to $F(z)$ on $E$ and the finite poles of the $r_{n v}(z)$ approach the $\nu$ poles of $F(z)$ in $T$. It then follows from the proof of Theorem 12 that there exists a function $f(z)$ meromorphic with at most $\nu$ poles in $E_{\rho}$ such that $f(z) \equiv F(z)$ for $z$ on $E$. Since $f(z)$ must be analytic on $E_{\rho}-T$ it is the desired continuation.

We conclude with an extension of [1, Theorem 3]:

THEOREM 13. With the geometric conditions of Lemma 2 suppose the function $f(z)$ is analytic on $\Gamma$ and is meromorphic with precisely $v$ poles in $E_{\rho}(\rho>1)$. Suppose $r_{n v}(z)$ is a sequence of rational functions of respective types $(n, v)$ which satisfy

$$
\limsup _{n \rightarrow \infty}\left[\max \left|f(z)-r_{n v}(z)\right| ; z \text { on } \Gamma\right]^{1 / n} \leqq 1 / \rho .
$$

Then for $n$ sufficiently large each $r_{n v}(z)$ has precisely $\nu$ finite poles, which approach respectively the $\nu$ poles of $f(z)$ in $E_{\rho}$; and the $r_{n v}(z)$ converge uniformly to $f(z)$ on each compact subset of $E_{\rho}$ which contains no pole of $f(z)$.

Theorem 13 generalizes [1, Theorem 3] since it does not assume that $f(z)$ is analytic on $E$. The proof of Theorem 13, which is left to the reader, follows from Theorem 5, Lemma 2, and the methods used in [1].

\section{REFERENCES}

1. J. L. Walsh, The convergence of sequences of rational functions of best approximation with some free poles, Proc. Sympos. Approximation of Functions, General Motors, Research Lab., 1964, Elsevier, Amsterdam.

2. —_, Interpolation and approximation, 3rd ed., Amer. Math. Soc. Colloq. Publ., Vol. 20, Amer. Math. Soc., Providence, R. I., 1960.

3. S. Warschawski, Bemerkung zu meiner Arbeit: Ubber die Randverhalten der Ableitung der Abbildungsfunktion bei konformer Abbildung, Math. Z. 38 (1934), 669-683. 
4. P. Dienes, The Taylor series, Dover, New York, 1957.

5. J. L. Walsh, Note on the convergence of approximating rational functions of prescribed type, Proc. Nat. Acad. Sci. U. S. A. 50 (1963), 791-794.

6. --, Approximation by bounded analytic functions, no. 144, Mémor. Sci. Math., Paris, 1960.

7. J. L. Walsh and H. M. Elliott, Polynomial approximation to harmonic and analytic functions: generalized continuity conditions, Trans. Amer. Math. Soc. 68 (1950), 183-203.

8. J. L. Walsh, An extension of the generalized Bernstein lemma, Colloq. Math. 16 (1967), 91-92.

\section{UNIVERSITY OF MARYLAND,}

Collegge Park, Maryland 